\title{
Novel Implementations for Microstrip Resonator Filters in Transversal and Alternative Topologies
}

\author{
David Cañete-Rebenaque ${ }^{1}$,Mónica Martínez-Mendoza ${ }^{1}$, Student Member, IEEE, J. \\ Pascual-García, Member, IEEE, J. S. Gómez Díaz, Student Member, IEEE, and \\ Alejandro Alvarez-Melcon ${ }^{1}$, Senior Member, IEEE,
}

\begin{abstract}
This paper presents novel and compact implementations of microstrip filters using transversal topologies. The structures proposed are based on source/load-multiresonator coupling, and implement different topologies, allowing the synthesis of different frequency responses with transmission zeros for maximum selectivity. Design guidelines for different resonator topologies are also reported to show the possibilities of the proposed structures. The analyzed characteristics of these filters, obtained with commercial full-wave computer-aided tools, show excellent agreement with synthesis results. Several filter prototypes of third order, showing different frequency responses, are manufactured and tested, obtaining good agreement with theoretical predictions.
\end{abstract}

Index Terms - Resonator filters, bandpass filters, microwave filters, planar filters, transversal filters.

\section{INTRODUCTION}

$\mathbf{S}$ YSTEMS based on microwave technology have an increasing impact in civil and consumer society. Modern communication systems such as satellite communications, radar or wireless systems, and the proliferation of portable devices, require more and more multiband applications and a better use of the electromagnetic spectrum. This requires the use of microwave filters with characteristics like multiband operation, sharp transition slopes or different transfer functions. In particular, the use of microwave filters with transmission zeros in certain frequencies are very convenient in these conditions. Multiple techniques and methodologies are available for designing microwave filters. Review papers as [1] or [2] show the state of the art in this field.

Recent contributions of microwave filters have been focused on the synthesis and design of elliptic or quasi-elliptic filters for channel separation or multiband structures [3], [4]. The insertion of transmission zeros at finite frequencies provides the necessary sharp cutoff slopes, depending on the requirements of the application. In particular, synthesis techniques available for the design of filtering structures, implementing transmission zeros, could be based in a main structure with an in-line coupling topology, to which additional bypass or cross coupling between nonadjacent resonators are introduced [5], [6].

Another possibility will be used in this paper. The source and the load will be connected directly to several resonators in a shunt configuration. In this way more than one main path to the signal between the input and output ports are

\footnotetext{
${ }^{1}$ Technical University of Cartagena, Campus Muralla del Mar s/n, Cartagena, E-30202, Spain, e-mail: david.canete@upct.es
}

provided. The transmission zeros will be a consequence of the destructive interference that occurs at the output port. Several coupling schemes and implementations using this technique were proposed in [7]. These are based on the concept of transversal filters first introduced in [8].

In this paper, we present different coupling schemes of filters with three resonant nodes and input-output multiresonator coupling, extending the basic topologies presented in [9]. Also note that practical implementations of transversal filers have recently been proposed in [10], [11]. In these works the filters were implemented using a hybrid waveguideprinted technology, where a microstrip line resonator was combined with the resonance of a partially filled dielectric loaded waveguide cavity. On the contrary, in the present work we propose practical implementations using a fully planar technology. The coupling schemes chosen, and the practical implementations using microstrip resonators, will allow for the synthesis of different frequency responses, showing the flexibility of the new configurations.

The most typical filter topology using three resonators is based on a trisection block, as shown in Fig. 1(a). Interesting examples of this topology can be found in [12], [13]. This basic structure allows the introduction of one transmission zero in the frequency response of the filter. Higher order filters can also be designed by cascading several trisections [14], [15]. One advantage of the alternative coupling schemes shown in Fig. 1(b)-(d), is the possibility to implement two transmission zeros in very compact configurations [16]. a)

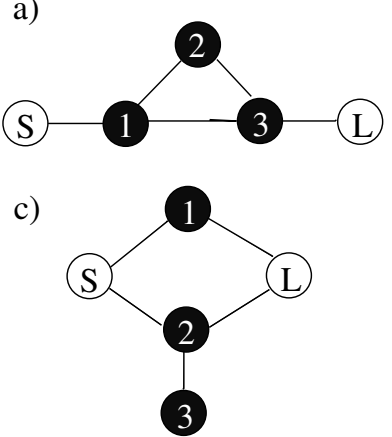

b)

d)

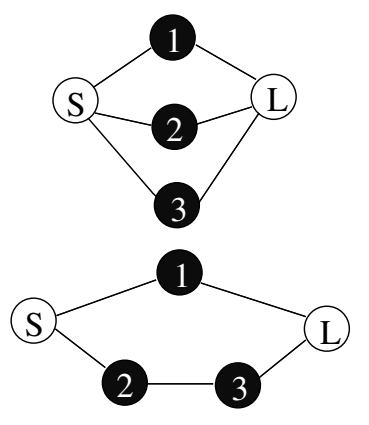

Fig. 1. Coupling schemes. (a) Single trisection. (b) Transversal third order topology. (c) and (d) Alternative three-resonator structures combining transversal and in-line sections.

This paper presents compact implementations in microstrip technology for the coupling schemes shown in Fig. 1(b)-(d). 
For the practical implementation, the resonators employed are $(\lambda / 2)$ and $(\lambda)$ microstrip printed lines. Several combinations of open-ended or short-circuited resonator lines are used, depending on the required signs of the coupling terms in the associated coupling matrices. We demonstrate that by changing the coupling signs and the resonator lengths appropriately, the positions of the transmission zeros can be conveniently controlled. Using this strategy, several frequency responses are possible, ranging from dual-band to quasi-elliptic responses. The modular design is another advantage of the proposed structures. The different resonators can be designed isolated, and when they are introduced in the final compact structure, only an easy optimization process to recover the final response is needed.

In addition to the theoretical discussion, several filter prototypes implementing different frequency responses are manufactured and tested. Measured results are found to be in good agreement with respect to predictions, therefore validating the new filtering structures.

\section{NOVEl Microstrip TOPOLOGIES}

The coupling and routing schemes of several third-order filters used in this paper are shown in Fig. 1. The dark circles represent resonators, the source and the load are shown as empty circles, and the solid lines represent coupling between nodes. The purpose of this section is to introduce new microstrip structures to implement these coupling schemes.

The first step in the design process of a filter is the calculation of the coupling matrix that synthesizes the desired frequency response. It should be noted that for the topologies presented in Fig. 1(b)-(d), a maximum of two transmission zeros at finite frequencies can be implemented. This can be inferred by the application of the minimum path rule [16]. For the pure transversal topology shown in Fig. 1(b), the coupling matrix can be easily calculated using the procedure presented in [8]. For multiband frequency responses, the procedure described in [3] can be used. On the contrary, for the topologies proposed in Fig. 1(c),(d), there are not direct synthesis techniques. One strategy in this case is to start from the transversal coupling matrix obtained from [8], and then apply rotations to annihilate the undesired coupling. When this is not possible, one can still resort to optimization techniques as reported in [17], [18].

Once the coupling matrix has been computed, the next step is to synthesize the required coupling with a suitable topology [19]. An advantage of the transversal schemes is that the different resonators of the structure can be designed independently. According to the coupling matrix values obtained during the synthesis procedure, the resonant frequencies of the resonators (given by the diagonal elements of the coupling matrix $M_{i i}$ ) and the external quality factors (related to the coupling from the resonators to the input/output ports $M_{S i}, M_{L i}$ ) are adjusted, and in this way the physical dimensions are obtained (coupling gaps and lengths of resonators) [20]. A practical example of a filter with dual-band operation and two transmission zeros, following the coupling topology of Fig. 1(b) was presented in [9] (Section II.A).
In the next subsections we present several new examples using alternative microstrip structures. These structures contain different kind of printed line resonators, and side-coupling are combined with broad-side coupling, when necessary, to implement the required coupling according to the proposed scheme (Fig. 1). To adjust the sign of the coupling, it is proposed to use resonators working at the first or second resonance when required.

\section{A. Transversal filter with quasi-elliptic frequency response}

The first proposed approach corresponds to a pure transversal topology (as shown in Fig. 1(b)), implementing a quasielliptic response. To place one transmission zero on both sides of the passband for maximum selectivity, only one negative coupling is required. The other two resonators should exhibit positive coupling to both ports.

To illustrate the concept, we now design a single-band filter with quasi-elliptic response centered at $1400 \mathrm{MHz}$ and a bandwidth of $100 \mathrm{MHz}$. The two transmission zeros are located at $1330 \mathrm{MHz}$ and $1470 \mathrm{MHz}$, and the ripple level is $20 \mathrm{~dB}$. Since the topology corresponds to a transversal filter of order three, the synthesis procedure reported in [8] can be directly used to obtain the coupling matrix

$$
M_{A}=\left(\begin{array}{rrrrr}
0 & 0.4471 & 0.4235 & -0.8647 & 0 \\
0.4471 & 1.3954 & 0 & 0 & 0.4471 \\
0.4235 & 0 & -1.3749 & 0 & 0.4235 \\
-0.8647 & 0 & 0 & -0.0452 & 0.8647 \\
0 & 0.4471 & 0.4235 & 0.8647 & 0
\end{array}\right)
$$

For the practical implementation of this filter we select a microstrip structure with two dielectric substrates broadside coupled, as shown in Fig. 2. In the first substrate we print two resonators, together with the input/output lines. In the substrate on top we print the remaining resonator. In this way we can obtain a pure transversal topology of order three, with a very compact microstrip structure.

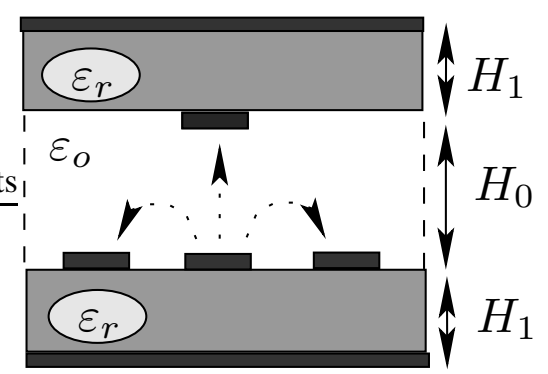

Fig. 2. Lateral view of the proposed filter containing two broadside coupled dielectric substrates.

A filter with similar frequency response was proposed in [9] (Section II.B). In that work, the two positive coupling signs were implemented using a meander-line resonator working at the $\lambda$-resonance and a short-circuited resonator. A drawback of this approach is the difficulty in the characterization of the via-hole used to implement the short circuit. Also, a small spurious radiation was observed due to the implementation of 
the via-hole. To overcome these problems, we propose now a new structure that maintains the coupling scheme and a similar quasi-elliptic response. To implement the required sign change, the short-circuited resonator is modified by introducing a meander type resonator that operates at the $\lambda$ resonance. With this structure, the spurious radiation of the short-circuited resonator is avoided at the expense of a longer resonator.To avoid direct coupling between resonators and to maintain the transversal topology, the positions of the resonators are also interchanged with respect to the topology given in [9]. In this case we propose to place the $\lambda / 2$ resonator on the upper substrate, so that coupling to adjacent resonators is minimized. With all these considerations, the new layout for the proposed microstrip configuration is shown in Fig. 3.

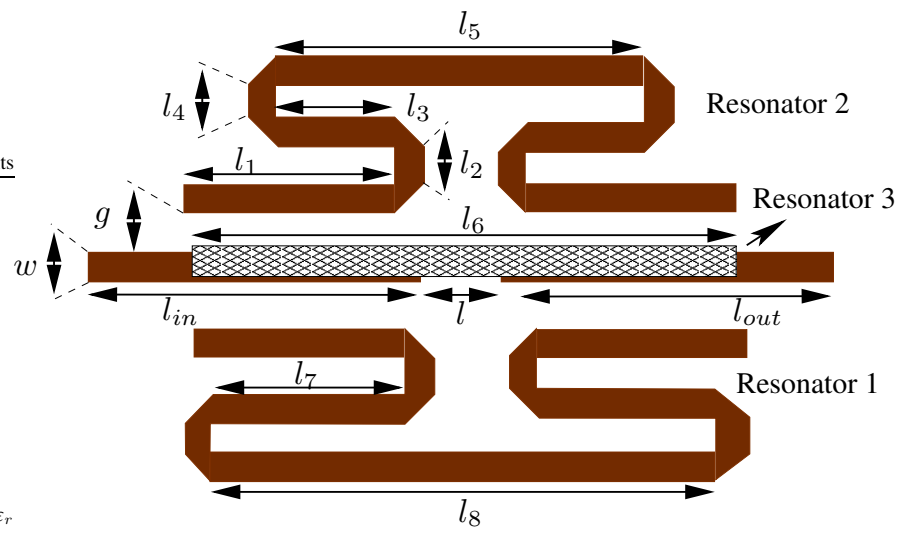

Fig. 3. Layout of the third order transversal filter with quasi-elliptic response. The $\lambda / 2$ resonator is printed in the upper substrate (see Fig. 2).

TABLE I

DIMENSIONS OF THE DESIGNED QUASI-ELLIPTIC FILTER SHOWN IN FIG. 3.

\begin{tabular}{|c|c||c|c|}
\hline Dimension & $\begin{array}{c}\text { Value } \\
\text { (in millimeters) }\end{array}$ & Dimension & $\begin{array}{c}\text { Value } \\
\text { (in millimeters) }\end{array}$ \\
\hline \hline$H_{0}$ & 1.0 & $l_{1}$ & 20.8 \\
$H_{1}$ & 1.27 & $l_{2}$ & 1.5 \\
$\varepsilon_{r}$ & 6.15 & $l_{3}$ & 13.2 \\
$w$ & 1.85 & $l_{4}$ & 1.5 \\
$g$ & 0.3 & $l_{5}$ & 26.0 \\
$l_{\text {in }}$ & 30.0 & $l_{6}$ & 49.6 \\
$l_{\text {out }}$ & 30.0 & $l_{7}$ & 45.4 \\
$l$ & 5.0 & $l_{8}$ & 1.5 \\
\hline
\end{tabular}

In Fig. 4 we show the results obtained with the coupling matrix and with fullwave simulations. The results agree very well, specially inside the passband. However, some discrepancies between the matrix and the full-wave approaches can be observed in the low stopband region of the filter. This is mainly due to a weak parasitic coupling between the input and output ports in the real structure. This additional parasitic coupling produces a third transmission zero below the passband, explaining the discrepancies. To demonstrate this, we also show in Fig. 4 the response of the coupling matrix when a small coupling is added between the input and output ports $\left(M_{S L}=M_{L S}=0.04\right)$.

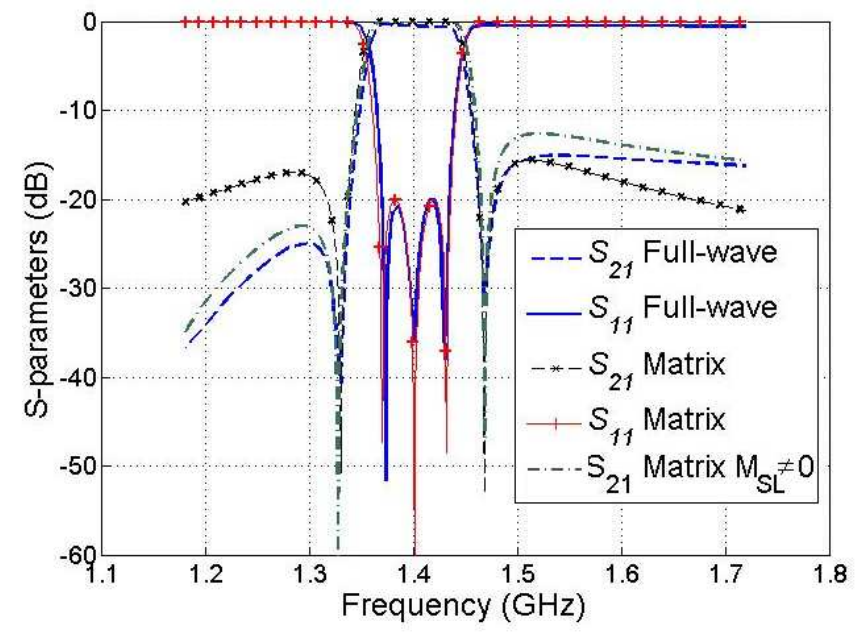

Fig. 4. Fullwave simulation and coupling matrix response of the quasielliptic transversal filter shown in Fig. 2 and Fig. 3. Results obtained with the coupling matrix with a small direct input/output coupling $\left(M_{S L} \neq 0\right)$ are also included to show the effects of parasitic coupling.

\section{B. Three-pole filter with transmission zeros in the complex plane}

The advantage of the topology explored so far, is that it implements pure transversal filters. Therefore, the calculation of the relevant coupling matrix can be obtained easily from well known synthesis techniques [8]. However, the disadvantage is that the broadside coupling structures introduced, complicate the manufacturing process. In this section we present an alternative filter configuration that only requires one dielectric substrate.

With one dielectric substrate, it is very difficult to couple the input/output ports to three resonators at the same time. For a single substrate microstrip configuration, it is better to use a different coupling scheme, as shown in Fig. 1(c). In this alternative configuration, the ports are coupled to only two resonators. An inter-resonator coupling is introduced from one of these resonators to the third resonator.

A direct synthesis technique to compute the coupling matrix of this structure is not available. However, we can start from the calculation of the coupling matrix of the transversal topology. By applying a series of similarity transformations, the equivalent folded canonical matrix [8] can be easily obtained. Then, a cross-pivot transformation to annihilate the element that represents the coupling between resonators 1 and 3 ( $M_{13}$, element $(2,4)$ of the coupling matrix) will automatically generate the coupling $M_{23}$, resulting into the desired coupling matrix.

As an example, we present the synthesis of a filter with two transmission zeros in the complex plane centered in the passband, used for equalization. The two transmission zeros are located at $1.45 \mathrm{GHz}$ (with real part $\delta=1.5$ ). The filter presents a total bandwidth of $100 \mathrm{MHz}$ and the ripple level is 
$15 \mathrm{~dB}$.

$$
M_{B}=\left(\begin{array}{rrrrr}
0 & 0.554 & -0.945 & 0 & 0 \\
0.554 & -0.243 & 0 & 0 & 0.554 \\
-0.945 & 0 & -0.299 & -1.319 & 0.945 \\
0 & 0 & -1.318 & 0.292 & 0 \\
0 & 0.554 & 0.945 & 0 & 0
\end{array}\right)
$$

From the matrix $M_{B}$ we can observe that the first resonator does not change sign from input to the output port. This can be obtained again with a meander line type microstrip resonator of length $(\lambda)$. The second resonator is again coupled to the input/output ports, but this time a sign change must be implemented. This can be obtained with a simple $(\lambda / 2)$ microstrip line resonator. Finally, this resonator is coupled to the third resonator. The most compact form to implement this coupling is through a standard coupled lines section, as shown in Fig. 5. In this structure the $l_{8}$ coupling gap is adjusted to achieve the required coupling between resonators 2 and 3 . The design procedure is again carried out by isolating the resonators one by one. For the resonators coupled directly to the ports, the relevant coupling matrix elements are again related to the external quality factor and to the resonant frequencies. For the two coupled resonators, the inter-resonator coupling is extracted under weak input/output coupling [20].

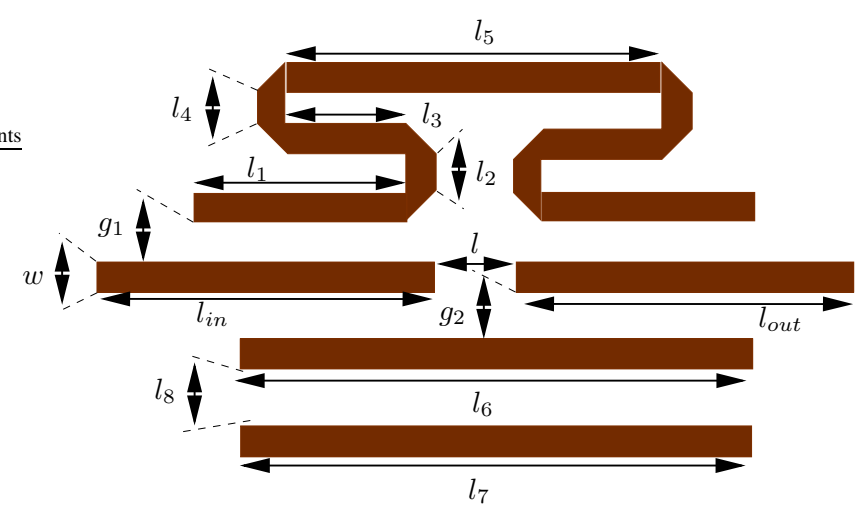

Fig. 5. Layout of a third order planar filter, implementing the coupling scheme shown in Fig. 1(c).

TABLE II

DIMENSIONS OF THE MANUFACTURED THIRD ORDER FILTER SHOWN IN FIG. 5.

\begin{tabular}{|c|c||c|c|}
\hline Dimension & $\begin{array}{c}\text { Value } \\
\text { (in millimeters) }\end{array}$ & Dimension & $\begin{array}{c}\text { Value } \\
\text { (in millimeters) }\end{array}$ \\
\hline \hline Substrate $H_{1}$ & 1.27 & $l_{1}$ & 19.6 \\
$\varepsilon_{r}$ & 6.15 & $l_{2}$ & 1.5 \\
$g_{1}$ & 0.3 & $l_{3}$ & 11.7 \\
$g_{2}$ & 0.5 & $l_{4}$ & 1.5 \\
$l_{\text {in }}=l_{\text {out }}$ & 30.0 & $l_{5}$ & 31.4 \\
$w$ & 1.85 & $l_{6}=l_{7}$ & 48.6 \\
$l$ & 5.0 & $l_{8}$ & 3.1 \\
\hline
\end{tabular}

The dimensions obtained for this filter are collected in Table II. In Fig. 6 we present the response of this structure, together with the results given by the coupling matrix $M_{B}$ (2). We can observe very good agreement between the two

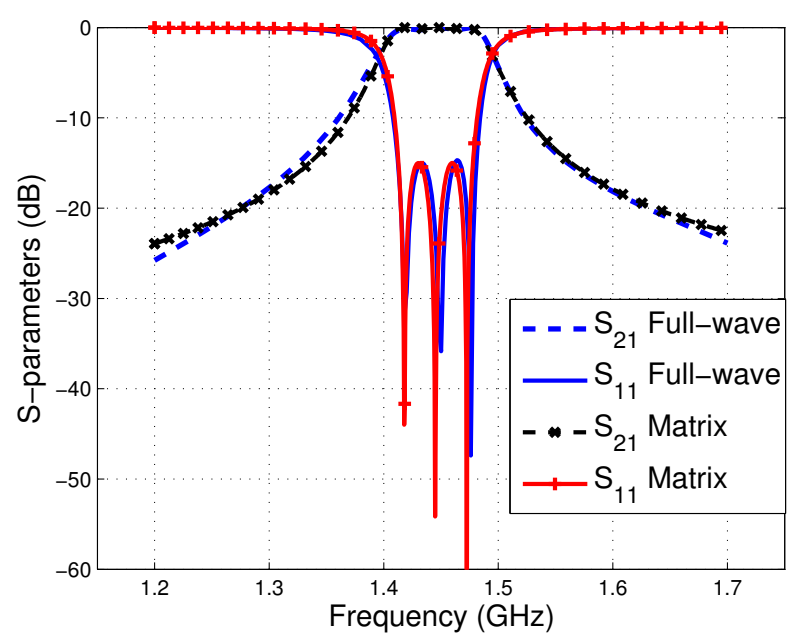

Fig. 6. Three pole filter design with two transmission zeros in the complex plane. Fullwave simulation and coupling matrix response for the topology shown in Fig. 5.

responses. Note the different type of response between this filter (with complex transmission zeros) and the quasi-elliptic filter shown in Section II.A; it is obvious the loss of selectivity. However, this filter exhibits less variations of the group delay within the passband. This is shown in Fig. 7, where we present the group delay of this filter compared to the group delay of a similar filter but with quasi-elliptic response. For the comparison, we have selected the same center frequency, bandwidth and return-loss levels for both filters.

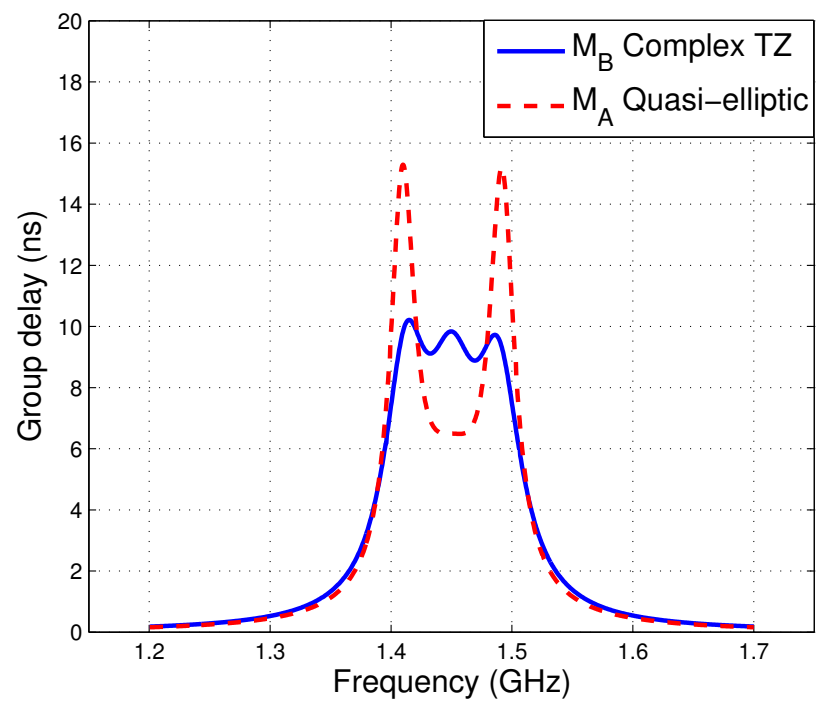

Fig. 7. Group delay response of filters $A$ ) and $B$ ).

The figure shows that the in-band variation of the group delay is greater for the quasi-elliptic filter $A)(8.80 \mathrm{~ns})$, than for the filter $B$ ) (only $2.05 \mathrm{~ns}$ ), thus confirming the expected trade-off between selectivity and group delay equalization. 


\section{Three-pole filter with two passbands and two transmission zeros}

Previous sections have shown different types of bandpass responses for microstrip filters (quasi-elliptic response or complex transmission zeros for phase equalization). This third example is expected to demonstrate that a dual bandpass response can be obtained with an alternative coupling topology. The advantage of this topology, with respect to the one presented in [9], is that only one dielectric substrate is needed, therefore simplifying the manufacturing process. In order to design a compact structure using only one dielectric substrate, the pure transversal topology introduced in Fig. 1(b) can be transformed into the alternative scheme shown in Fig. 1(d).

As an example, we propose to design a similar dualbandpass filter as the one studied in Section II-A of [9]. In this case the filter has a center frequency of $1.4 \mathrm{GHz}$, with two transmission zeros $(1.37 \mathrm{GHz}$ and $1.62 \mathrm{GHz})$. The lower passband has a bandwidth of $20 \mathrm{MHz}$ while the bandwidth of the upper passband is $80 \mathrm{MHz}$. Finally, the ripple level is $-17 \mathrm{~dB}$. For the synthesis of a coupling matrix describing this topology, we use the procedure reported in [3] for multiband responses, combined with an optimization technique described in [17]. According to this procedure, the following coupling matrix is obtained

$$
M_{C}=\left(\begin{array}{rrrrr}
0 & 0.314 & -0.598 & 0 & 0 \\
0.314 & 0.870 & 0 & 0 & 0.314 \\
-0.598 & 0 & -0.586 & 0.445 & 0 \\
0 & 0 & 0.445 & -0.586 & -0.598 \\
0 & 0.314 & 0 & -0.598 & 0
\end{array}\right)
$$

For the practical implementation of this coupling matrix, we propose to use the structure shown in Fig. 8. As shown

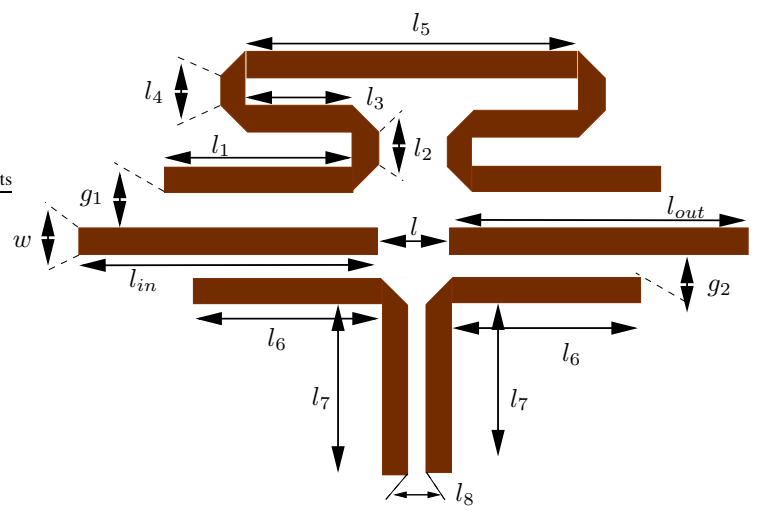

Fig. 8. Layout of a third order transversal microstrip filter, implementing the coupling scheme shown in Fig. 1(d).

in Fig. 1(d), the first resonator must be coupled to the input and output ports simultaneously. From the second column of the matrix $M_{C}$ (3), we can observe that the same sign must be applied to both coupling elements. To implement this, a meander line resonator of length $(\lambda)$ is again used, as shown in Fig. 8. The remaining negative coupling terms shown in the matrix $M_{C}$ can be implemented by using printed line resonators of length $(\lambda / 2)$. In this case, a single $90^{\circ}$ bend is included in the $(\lambda / 2)$ resonators, to allow for the introduction of an inter-resonator coupling between the second and third resonators (see Fig. 1(d) and Fig. 8).

The design process for this filter is similar to the one described before. Each resonator is adjusted separately. The meander resonator is coupled directly to the ports, and the relevant coupling matrix elements are related to its resonant frequency (the diagonal term $M_{11}$ ) and to the external quality factor (coupling $M_{S 1}=M_{L 1}$ controlled by the gap $g_{1}$ ). The next step is the design of the bended microstrip line resonators. The resonant frequency is again related to the diagonal terms of the coupling matrix $\left(M_{22}=M_{33}\right)$, while the coupling to the ports is related to the external quality factor (couplings $M_{S 2}=M_{L 3}$ controlled by the gap $g_{2}$ ). Finally, the inter-resonator coupling is obtained under weak input/output coupling as explained in [20] (coupling $M_{23}$ controlled by the gap $l_{8}$ ).

In Table III we show the final dimensions of the designed filter. The electrical response of this structure is shown in

TABLE III

DIMENSIONS OF THE MANUFACTURED DUAL-BANDPASS FILTER SHOWN IN FIG. 8.

\begin{tabular}{|c|c||c|c|}
\hline Dimension & $\begin{array}{c}\text { Value } \\
\text { (in millimeters) }\end{array}$ & Dimension & $\begin{array}{c}\text { Value } \\
\text { (in millimeters) }\end{array}$ \\
\hline \hline Substrate $H_{1}$ & 1.27 & $l_{1}$ & 20.8 \\
$\varepsilon_{r}$ & 6.15 & $l_{2}=l_{4}$ & 1.5 \\
$g_{1}$ & 0.3 & $l_{3}$ & 13.2 \\
$g_{2}$ & 0.4 & $l_{5}$ & 35.0 \\
$l_{\text {in }}=l_{\text {out }}$ & 30.0 & $l_{6}$ & 23.3 \\
$w$ & 1.85 & $l_{7}$ & 25.2 \\
$l$ & 5.0 & $l_{8}$ & 0.4 \\
\hline
\end{tabular}

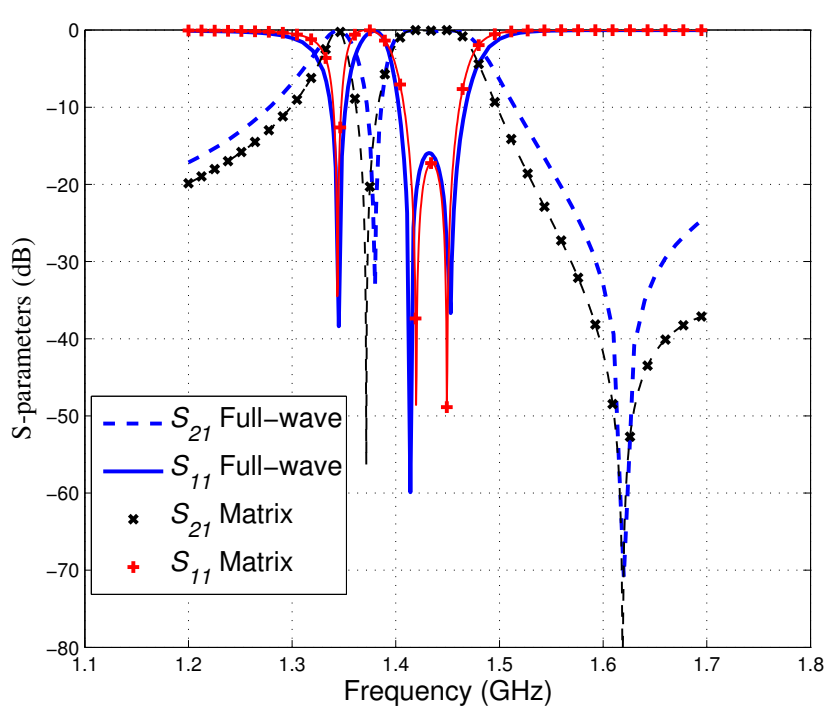

Fig. 9. Three pole filter design for a dual-bandpass response. Fullwave simulation and coupling matrix response for the topology shown in Fig. 8.

Fig. 9, together with the results obtained from the analysis of the coupling matrix $M_{C}$ (3). We can again observe good agreement between the two responses. 


\section{RESULTS AND EXPERIMENTAL IMPLEMENTATION}

For a practical validation of the filter designs presented in this paper, several prototypes have been manufactured and tested in microstrip technology. The basic layouts with the relevant dimensions of the fabricated filters are shown in Fig. 3, Fig. 5 and Fig. 8. The substrate selected for manufacturing is an RT-Duroid/6006, with a relative permittivity $\varepsilon_{r}=6.15$, and thickness $1.27 \mathrm{~mm}$ ( $H_{1}$ in Fig. 2). The data obtained from fullwave simulation in this section consider dielectric losses $(\tan (\delta)=0.0019)$ and losses in the printed metalizations $\left(\sigma=4 \cdot 10^{7} \Omega^{-1} / \mathrm{m}\right)$.

In Fig. 10 we present the simulated results for the transversal filter shown in Fig. 3. This filter implements a pure transversal topology of order three, with two transmission zeros on both sides of the passband (first example of the paper).

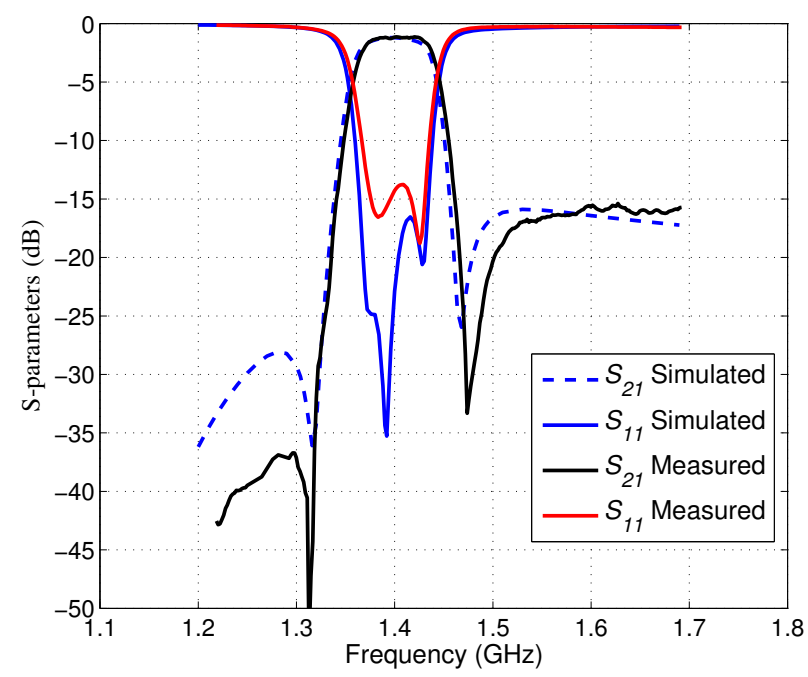

Fig. 10. Comparison between simulated and measured results for the filter shown in Fig. 3.

Measurements show good agreement with theoretical predictions. The measurements indicate insertion losses of $1.2 \mathrm{~dB}$, return losses of $13.8 \mathrm{~dB}$, and a bandwidth of $80 \mathrm{MHz}$. This manufactured prototype is shown in Fig. 11. The basic structure consists of two stacked dielectric substrates separated by a layer of air. The substrates are properly aligned to allow for a precise adjustment of the broadside coupling.

The next example implements the coupling scheme shown in Fig. 1(c), using the layout presented in Fig. 5. In this case, the two transmission zeros are placed in the complex plane for phase equalization. In Fig. 12 we present the measured results for this filter, and we compare them with results obtained from the analysis of the structure. We can observe fair agreement between the two responses. The minimum insertion losses of the filter within the passband is $2.3 \mathrm{~dB}$. The level of ripple obtained is $-16.6 \mathrm{~dB}$, while the bandwidth is $110 \mathrm{MHz}$.

Finally, a third example has been manufactured and tested in order to provide experimental verification for the topology shown in Fig. 1(d). To implement this topology, the layout presented in Fig. 8 has been used. In Fig. 13 we include the

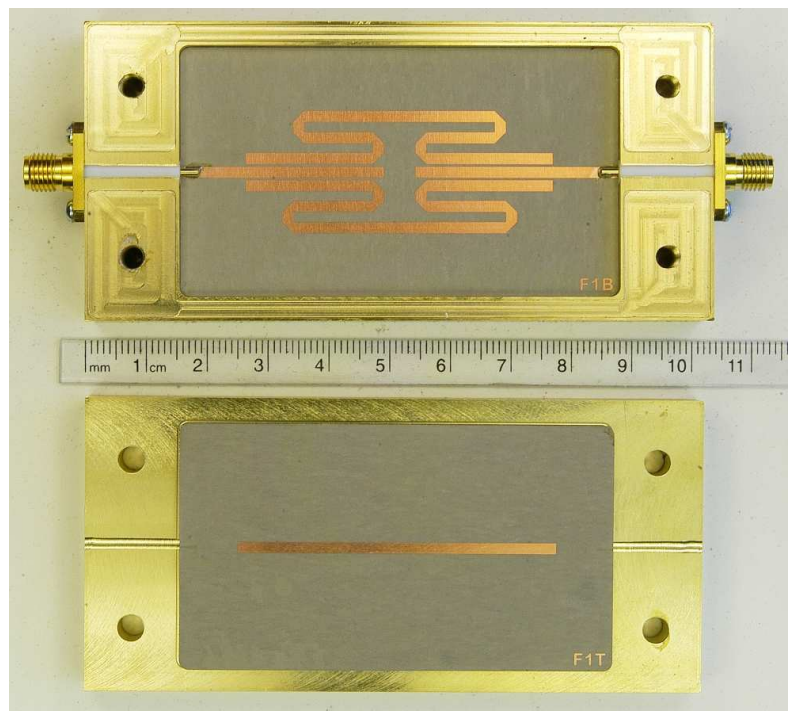

Fig. 11. Photo of the first manufactured prototype.

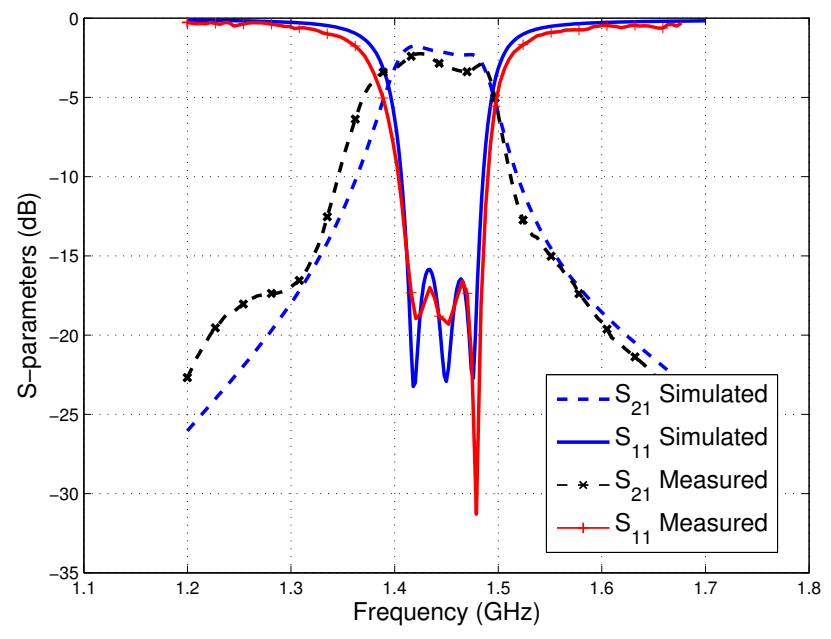

Fig. 12. Comparison between simulated and measured results for the filter shown in Fig. 5.

measured results, and we compare them against the response obtained from the analysis of the structure. It can be observed that the simulated and measured results are in fair agreement. The minimum insertion loss of the filter inside the lower passband is $3.2 \mathrm{~dB}$, whereas inside the upper passband the minimum insertion loss decreases to $2.6 \mathrm{~dB}$. The two last measured results exhibit higher insertion losses than the simulated responses. This is due to worse manufacturing tolerances than the first prototype. There are also slight radiation losses, since these two filters are not shielded.

These last planar circuits have been manufactured with an LPKF Protomat drilling machine, and measurements are taken with an E5071B vector network analyzer. A photograph of the two last manufactured prototypes is presented in Fig. 14. In these two cases, the whole filters are implemented using only one dielectric substrate. This simplifies the manufacturing process with respect to the structures presented in [9], since 


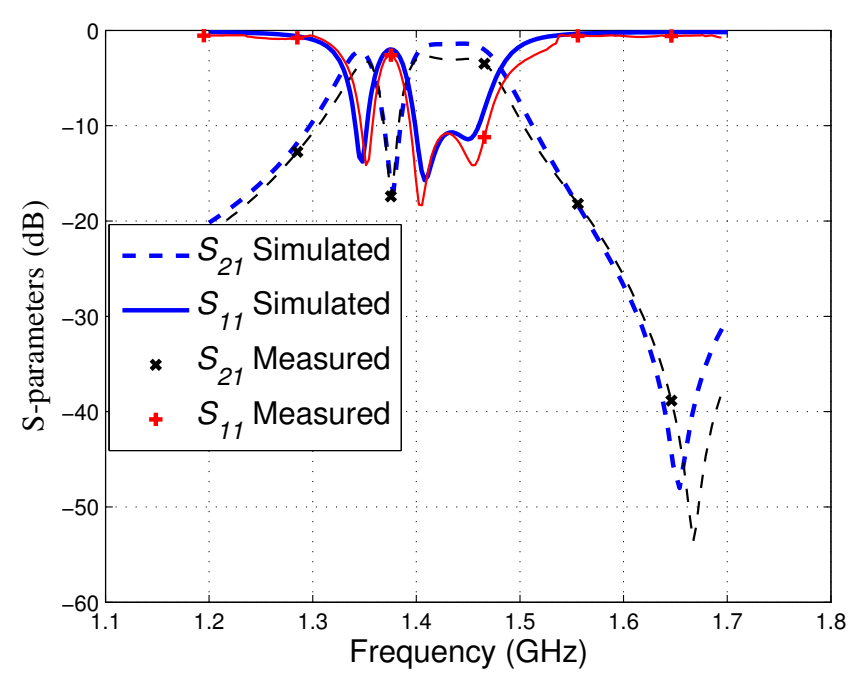

Fig. 13. Comparison between simulated and measured results for the filter shown in Fig. 8.

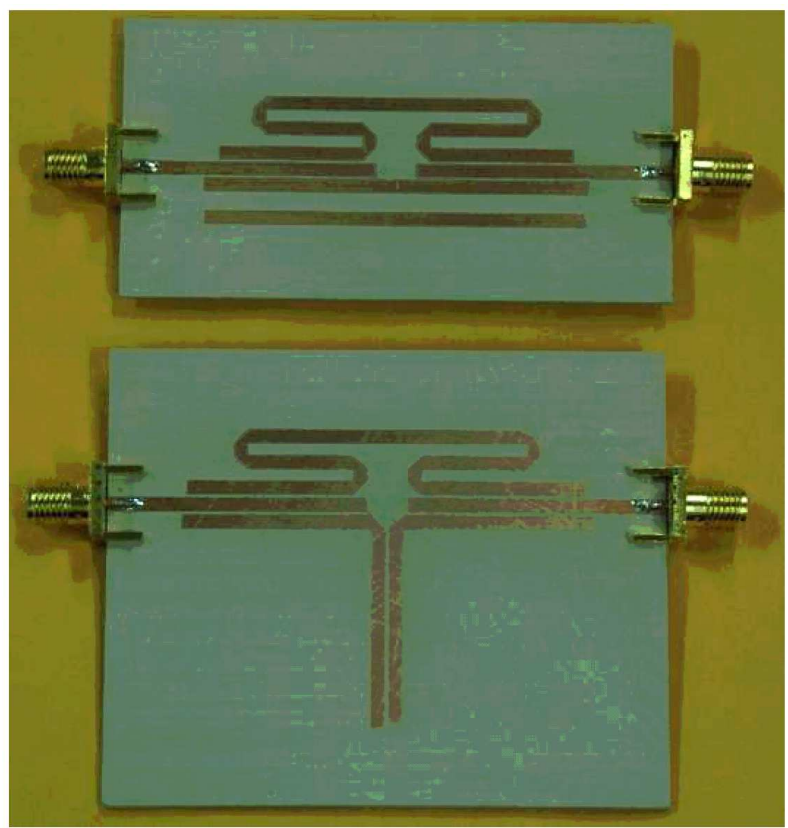

Fig. 14. Photo of the two last manufactured prototypes.

alignment operations between different dielectric layers are avoided.

\section{CONCLUSIONS}

Several third-order novel microstrip bandpass filters have been designed, manufactured and tested. Three coupling schemes of third-order filters, exhibiting two transmission zeros, have been implemented in microstrip technology. It is shown that several frequency responses can be implemented, such as dual-band, quasi-elliptic, or with transmission zeros in the complex plane for phase equalization. Several strategies to adjust the coupling signs have been proposed, including the use of resonators of different lengths. The obtained measurements present good agreement with respect to theoretical predictions, showing the validity of the proposed structures.

\section{REFERENCES}

[1] R. Levy and S. B. Cohn, "A history of microwave filter research, design and development," IEEE Transactions on Microwave Theory and Techniques, vol. 32, pp. 1050-1067, Sept. 1984.

[2] R. Levy, R. V. Snyder, and G. Matthaei, "Design of microwave filters," IEEE Transactions on Microwave Theory and Techniques, vol. 50, pp. 783-793, March 2002.

[3] M. Mokhtaari, J. Bornemann, K. Rambabu, and S. Amari, "Couplingmatrix design of dual and triple passband filters," IEEE Transactions on Microwave Theory and Techniques, vol. 54, no. 11, pp. 3940-3946, November 2006.

[4] G. Macchiarella and S. Tamiazzo, "Design techniques for dual-passband filters," IEEE Transactions on Microwave Theory and Techniques, vol. 53, no. 11, pp. 3265-3271, Nobember 2005.

[5] R. M. Kurzok, "General four-resonator filters at microwave frequencies," IEEE Transactions on Microwave Theory and Techniques, vol. 14, no. 7, pp. 295-296, July 1966.

[6] J. S. Hong and M. J. Lancaster, "Design of highly selective microstrip bandpass filters with a single pair of attenuation poles at finite frequencies," IEEE Transactions on Microwave Theory and Techniques, vol. 48, no. 7, pp. 1098-1107, July 2000.

[7] U. Rosenberg and S. Amari, "Novel coupling schemes for microwave resonator filters," IEEE Transactions on Microwave Theory and Techniques, vol. 50, no. 12, pp. 2896-2902, December 2002.

[8] R. J. Cameron, "Advanced coupling matrix synthesis techniques for microwave filters," IEEE Transactions on Microwave Theory and Techniques, vol. 51, no. 1, pp. 1-10, January 2003.

[9] D. C. Rebenaque, M. M. Mendoza, J. P. García, J. S. G. Diaz, and A. A. Melcón, "Novel implementations for microstrip resonator filter in transversal topology," in European Microwave Conference. Rome, Italy: IEEE, 28 September-2 October 2009.

[10] M. Martínez-Mendoza, J. Gómez-Diaz, D. Canete-Rebenaque, J. Gómez-Tornero, and A. Álvarez-Melcón, "Design of bandpass transversal filters employing a novel hibrid structure," IEEE Transactions on Microwave Theory and Techniques, vol. 55, no. 12, Part 2, pp. 2670-2678, December 2007.

[11] M. Martínez-Mendoza, J. Gómez-Diaz, D. Canete-Rebenaque, and A. Álvarez-Melcón, "Design of dual-bandpass hybrid waveguidemicrostrip microwave filters," IEEE Transactions on Microwave Theory and Techniques, vol. 56, no. 12, pp. 2913-2920, December 2008.

[12] J. S. Hong and M. J. Lancaster, "Microstrip cross-coupled trisection bandpass filters with asymetric frequency characteristics," IEE Proceedings - Microwaves, Antennas and Propagation, vol. 146, no. 1, pp. 84 90, February 1999

[13] C. C. Yang and C. Y. Chang, "Microstrip cascade trisection filter," IEEE Microwave and Guided Wave Letters, vol. 9, no. 7, pp. 271-273, July 1999.

[14] J. T. M. Garay, "Synthesis of physically asymmetrical n-trisection filters with transmission zeros at n differente real frequencies," Electronics Letters, vol. 35, pp. 226-227, February 1999.

[15] G. Machiarella, "A powerfull tool for the synthesis of prototype filters with arbitrary topology," in Proceedings of IEEE, International Microwave Symposium. MTT-S, June 2003, vol. 3, pp. 1467-1470.

[16] S. Amari and J. Bornemann, "Maximum number of finite transmission zeros of coupled resonator filters with source/load-multiresonator coupling and a given topoloy," in Proceedings of Asia-Pacific Microwave Conference, Sydney, Australia, 3-6 Dec. 2000, pp. 1175-1177.

[17] S. Amari, U. Rosenberg, and J. Borneman, "Adaptative synthesis and design of resonator filters with source/load-multiresonator coupling," IEEE Transactions on Microwave Theory and Techniques, vol. 50, no. 8, pp. 1969-1978, August 2002.

[18] F. Seyfert and S. Bila, "General synthesis techniques for coupled resonator networks," IEEE Microwave Magazine, pp. 98-104, October 2007.

[19] D. Swanson and G. Macchiarella, "Microwave filter design by synthesis and optimization,” IEEE Microwave Magazine, pp. 57-69, April 2007.

[20] J.-S. Hong and M. J. Lancaster, Microstrip filters for RF/Microwave applications. John Wiley and Sons, 2001. 